\title{
Towards Personalized Elevator Travel with Smart Elevator System
}

\author{
Tarmo ROBAL, Uljana REINSALU, Mairo LEIER \\ Department of Computer Systems, Tallinn University of Technology, 12618 Tallinn, Estonia \\ \{tarmo.robal, uljana.reinsalu, mairo.leier\}@taltech.ee
}

\begin{abstract}
For ages, machines have been used to lift materials and goods. With the appearance of elevators on the 19th century, vertical transportation of people became efficiently available. The general concept of modern elevators, which became widely exploitable some 150 years ago, has regardless of many technological advancements remained mostly the same. The typical elevator still needs traveller's input to take the passenger from one floor to another. With innovation in technology, Cyber-Physical Social Systems (CPSS), where highly computerized systems work in tight connection with human users, have appeared. Smart elevators are a kind of CPSS. In this paper, we explore elevator travellers' behaviour and travel needs, and the possibility to predict passenger destination floor. For this, we use passenger profiles established through deep learning, and elaborate on their travel. The study is based on a real smart elevator system set up in a typical office building.
\end{abstract}

Keywords: smart elevator, floor prediction, travel personalization, travel profiling, CPS, cyberphysical social system (CPSS), machine learning, neural network.

\section{Introduction}

The history of modern elevators goes back to 19th century, although mechanisms for vertical transportation have existed even for a longer term. Elevators are used to meet the vertical transportation needs of people (and goods) in buildings. The domain today is regulated by several standards. The ISO 8100 standard series describe the standards for elevators, where the requirements for the transportation of persons and goods using elevators have been described by the ISO 8100-32 standard (ISO, 2020), and in addition the lift ride quality during lift motion by the ISO 18738 (ISO, 2012) standard. Since the advancement in engineering has enabled to establish ever higher buildings, elevators have become an important mean of vertical transportation, allowing people to move from one floor to another quickly and without any extra physical effort. In modern buildings today - either commercial, or residential - elevators are a necessity many people rely on, especially in mid- and high-rise buildings, as climbing several 
floors is tiring, and in some cases even impossible, e.g., elderly people and people with disabilities.

Most of today's elevators are operated manually from inside the elevator car by its passengers by selecting the desired destination floor from the switch panel. This is a simple, yet efficient approach, and provides a lot of liberty to the passengers. However, once a destination is set, it cannot be cancelled. The main problem of conventional elevators is that the passenger destination floor is unknown until the passenger has boarded the elevator car. This issue has been addressed by some more advanced elevator systems, which allow to pre-register destination floor together with an elevator call (Hikita et al., 2004), providing also a way to group and schedule the passengers moving to the same floor.

With the advancement of technology and overall computerization, Cyber Physical Systems (CPS) (Lee and Seshia, 2016) and Cyber Physical Social Systems (CPSS) (Cassandras, 2016; Dressler, 2018; Zhuge, 2014), emphasizing the crucial role of humans in highly computerized systems, have gained an importance in our lives, and leading the way to smart cities. Cities have always tried to be 'smarter' through the use of 'new technology', and elevators have also played a role there - enabling for example the development of skyscrapers (Gath-Morad et al., 2017). Today, this 'new technology' is information and communication technology (ICT), which has demonstrated an explosive growth in Artificial Intelligence (AI) technologies (Russel and Norvig, 2009) such as facial image recognition (Robal et al., 2018; Silva et al., 2018; Stark, 2019, Zhao et al., 2003) and human speech recognition (Allen, 2003; Goetsu and Sakai, 2019; Ketkar and Mukherjee, 2011; Ross et al., 2004), delivering a strong basis and technological advancement for establishing smart systems, such as self-service border control, selfdriving cars, and smart elevators (Bharti et al., 2017; Ge et al., 2018a; Ge et al., 2018b; Skog et al., 2017). A smart elevator is a CPSS advantaging of data mining, AI, and machine learning to enhance and ease the exploitation of elevator systems, and deliver personalized travel experience to its passengers.

Although, a lot of works can be found on CPSS, smart elevators as CPSS have received little attention. Most of the research around elevators falls into the scope of reducing waiting time for elevators and optimizing the energy consumption of elevators (Bamunuarachchi and Ranasinghe, 2015; Bharti et al., 2017; Chou et al., 2018; Fernandez and Cortes, 2015; Fujimura et al., 2013; Wang et al., 2011), the latter being an emerging focus since there is a strong need to reduce carbon footprint of human kind. Optimal parking in group elevator control was explored by Brand and Nikovski (2004), whereas the use of floor sensors and RFID for elevator scheduling was of interest for Kwon and colleagues (2014), and the use of mobile phones to improve flow of people for Turunen et al. (2013). Optimization of elevator evacuation in smart high-rise buildings was explored by Ding et al. (2014). Thorough overview of elevator control systems is provided in the work of Fernandez and Cortes (2015). Intelligent context-aware elevator systems have been studied by Ge et al. (2018a), and passenger behavioral patterns while using elevators by Liang et al. (2013).

The majority of the prior research work on elevator scheduling systems has explored possibilities of using genetic algorithms (Kim and Moon, 2001) and genetic networks (Eguchi et al., 2004), artificial neural networks (Dewen et al., 1997), and DNA comput- 
ing methods (Zhao and Liu., 2013), or exploiting the context of elevator usage (Ge et al., 2018a, 2018b; Strang and Bauer, 2007). In these works the passengers are mostly addressed as anonymous units, and there exists no prior knowledge about the passengers or their preferences in the system. In our work, on the contrary, we are addressing elevator passengers about whom the smart elevator system has some prior knowledge stored and available for for making decisions. Thus, with our smart elevator system we are aiming to deliver a personalized travel experience for elevator passengers, and this distinguishes our work from prior existing research.

Providing personalized services has been a research ground for many studies, mostly facilitating from systems of information retrieval (IR), extraction of user context, modelling and establishing user profiles (Gaudioso and Boticario, 2003; Eirinaki and Vazirgiannis 2003), re-ranking items and providing recommendations (Adomavicius and Tuzhilin, 2005; Brocken et al., 2019; Gauch et al., 2003; Robal and Kalja, 2010; Sieg et al., 2007; Speretta and Gauch, 2005), e.g. personalized news recommendations or Web personalization, delivering significant improvement to services provided. In this article, we take this approach towards CPSS, in particular to a smart elevator system.

Herein, our main focus is on elevator passengers for whom a user profile has been established (discussed in Section 3.3) in the system, and some travel history is available. For passengers who remain unidentified by the system, the travel with the smart elevator remains conventional, and the smart elevator floor prediction system does not provide any prediction nor floor pre-selection for the passenger. The utmost aim is to provide a personalized service for smart elevator passengers.

The elevator system we address in this paper is a single car single shaft elevator system run in a typical eight-floor office building. The building resides at a university campus and hosts both offices for private companies and university staff, and rooms for teaching (i.e., lecture halls, labs, and computer classes). The building has two independent elevator systems, out of which the North elevator has been equipped with features of a smart elevator, e.g., cameras, sensors, speakers, microphones, etc., and besides providing vertical transportation for building inhabitants and visitors, servers also as a smart elevator test-bed.

The objectives of the research described in this paper are to investigate elevator passengers' travel behaviour, and establish a baseline for floor prediction. To accomplish this, data about travels with a smart elevator is collected with actual travel information. To better understand elevator passengers travel needs and provide personalized travel experience, we also explore whether traveller behaviour data can be used to extract some travel patterns and apply these to improve destination floor predictions. These objectives take us to the following research questions to be studied:

RQ1: How likely are elevator travellers willing to take the elevator to travel just one floor? We hypothesize that the majority of people prefer to take stairs to transit one floor, as it is not a big effort and usually the elevator waiting time exceeds this effort time.

RQ2: To what extent does the most frequently travelled floor describes elevator passenger behaviour and travel needs? To answer this question, we look at passengers' travels and compare those to the baseline prediction based on the most probable floor within passenger travel history. 
RQ3: How dependent are elevator passengers' travel needs on their daily activities in a typical office building, and can this be used to improve floor prediction for a profiled passenger? To answer this question, we explore passengers daily travel patterns and include them to floor prediction. We hypothesize that including these daily travel patterns will improve prediction accuracy for profiled elevator passengers in a typical office building.

RQ4: What of existing machine learning algorithms could be possibly applied in the smart elevator system for floor prediction? To answer this question, we explore and experiment with the perceptron algorithm, logistic regression, $k$-nearest-neighbour, support vector machine, and decision tree approaches, and use them to simulate floor predictions for profiled passengers.

The results of our studies indicate that potential travel distance, as well as frequently travelled floors and the travel time are important attributes of smart elevator passenger profile, and serve as a valuable input for our future studies. The elevator travel need prediction is a non-linear problem, for which k-nearest neighbour and decision tree approaches promise to provide best results. This paper is an extended version of already published conference paper (14th Intl. Baltic Conference on Databases and Information Systems, Baltic DB\&IS 2020) on the same topic (Reinsalu et al., 2020).

The rest of the paper is organized as follows. In Section 2 we address related works in the domain of elevators and predicting user interaction and preferences, while in Section 3 we focus on the smart elevator system. Section 4 is dedicated for personalized elevator travel, and delivers the answers for the set four research questions. Finally, in Section 5 we draw conclusions and discuss ideas for future work.

\section{Related works}

The research on elevators has mainly focused on dispatch problems and alleviating the waiting time problem. Bharti et al. (2017) established a cognitive model for dispatch systems like smart elevators to reduce wait time, and predict a necessity of an elevator on a particular floor using machine learning. Their solution could be applied to other dispatch systems like bus and train to maximize the utilization of provided services. In their system travellers were identified using ID card's location, and persons were registered within the system with their regular destination floor. These two pieces of data were used to minimize elevator waiting time by making an automatic call to elevator based upon prediction of user's arrival into elevator area. Fujimura et al. (2013) on the other hand explored a similar problem of reducing passenger waiting time in the context of multi-car elevators in single shaft for high-rise buildings. Their control algorithm allocated requests to elevator cars by using a local search method to reduce the waste time during which cars cannot transport passengers. The algorithm was evaluated by simulation and showed improved performance compared to earlier algorithms used for multi-car elevators. Luo et al. (2005) described elevator traffic flow as a time series set, and predicted the traffic using the least squares support vector machines, providing promising results and good performance.

While several researchers address controlling the elevator for optimized usage, others have focused on monitoring elevator behaviour for failure detection and mainte- 
nance. Skog et al. (2017) investigated the possibility of using a sensor node 'blackbox' unit to cost-efficiently modernize existing elevator systems and enable condition monitoring capabilities in such systems using non-intrusive sensing via accelerometers and magnetometers, embedded data processing, and wireless connectivity. Their experiments showed that using low-cost sensors similar to those found in smartphones, elevator position can be successfully estimated, and door closing time used to detect one of the most frequent faults of elevator systems - malfunctioning doors. Marinov et al. (2020) also explored engineering challenges for developing wireless sensor node for remote elevator condition monitoring. Their autonomous device contained accelerometer, magnetometer, and barometer to collect data about elevator position, quality of movement, vibration, and to detect elevator door malfunctions, similar to the setup of Skog et al. (2017). Hou et al. (2020) analyzed existing failure prediction methods, and constructed a knowledge graph of failures and proposed a method to improve failure prediction to cut maintenance cost and increase its quality. Olalere et al. (2018) on the other hand turned to and applied the concept of Internet of Things (IoT) to monitor elevator vibration and acoustics to enhance fault diagnostics and faster maintenance of elevators. Yaman and Karakose (2017) applied image processing and auto correlation, and proposed a method for monitoring elevator rope in real time for rope failure - which can have catastrophic results - detection.

Cameras and deep learning were used by Chou et al. (2018) not only to minimize the average waiting time for passengers but also to decrease energy consumption by re-scheduling elevator movements. In this research cameras were placed in front of the elevators and Region Based Convolutional Neural Network (R-CNN) was used to detect the number of passengers queuing for an elevator, and dispatch elevators according to the detected demand such that the elevator with the smallest energy consumption was serving the waiting passengers.

Ge et al. (2018a) took the concept of a smart elevator even further by turning a conventional elevator system into an IoT based elevator system, and proposed Intellevator - an intelligent elevator system able to proactively provide passengers assistive information (e.g., real-time waiting time) based on real-time context sensing and awareness. Passengers perceived Intellevator as useful. In parallel, another smart elevator system was proposed by Ge et al. (2018b) - a pre-call enabled elevator system in smart building called the PrecaElevator, where elevator could be pre-called within office room using a special user interface, reducing significantly passenger waiting times on a conventional office elevator. While Ge and colleagues relied on IoT, Strang and Bauer (2007) applied context-awareness to elevator system by exploiting available contextual knowledge (e.g., weather vs lunch-time, upcoming events in the building, etc), and made it available for control systems for elevator scheduling. Their work indicated that including the context can improve system performance.

While Ge et al. (2018b) allowed passengers to pre-call the PrecaElevator elevator from their office, Turunen et al. (2013) explored a novel concept of using mobile interaction with elevators. They allowed passengers to indicate in advance where they are going through a special smartphone app, whereas the application handled the corresponding elevator calls on behalf of them. The app also allowed to define default departure and destination floors. The important finding of this study is that mobile apps 
can be used to place elevator calls in advance, and it can shorten the perceived waiting time and expedite the movement of passengers in buildings.

With the advancement of ICT, voice user interfaces (VUI) (Pearl, 2017) are finding their way into many systems, for instance smartphones (e.g., Apple Siri) and smart speakers (e.g. Amazon Echo Dot, Google Home), but have also gained the interest of researchers in the context of elevators, as an alternative to common touch-button interface. Cernys et al. (2003) used voice recognition in an intelligent lift control model using a modification of the Dynamic Time Warping (DTW) algorithm. The setup of their system contained only eight Lithuanian words as commands, and for this simple setup they achieved voice recognition accuracy almost equal to $100 \%$. Cernys et al. (2003) argue that elevators can be successfully controlled by a small set of voice commands; the most important is to have high recognition accuracy of voice. The authors also conclude that voice controlled systems are especially useful for disabled people. Voice control option of an elevator is also found attractive by Meenatchi et al. (2015), who studied a voice-controlled simulated elevator system for disabled people, and also included a voice feedback system for cases of emergency. They were able to gain voice recognition accuracy of at least $90 \%$ for their system setup.

Human behavioural patterns in elevator usage were investigated by Liang et al. (2013). Based on real-world traces collected from 12 elevators in an 18-story office building, they argue that understanding human behavioral patterns is an important step towards better elevator usage efficiency, and behavioral patterns affect the service the most in high-rise buildings with several elevators, as in low-rise buildings the elevators can reach all floors quickly and the effect of service time therefore is rather small.

Personalizing services, predicting items of interest and recommending those, has been researched mostly in connection of e-commerce and Semantic Web. Daily we use Web browsers which have built-in prediction features for addresses we might want to visit, or data to fill in forms, without us giving much of a thought of it. Eirinaki and Vazirgiannis (2003) applied statistical and data mining methods to Web log data in order to extract useful patterns of Web users' navigational behavior to personalize Web experience and customize a Web site to the needs of specific users. Brocken et al. (2019) and van Huijsduijnen L.H. et al. (2020) applied natural language processing and Semantic Web methods to predict users' interests and recommend news items. Robal and Kalja (2010) established a conceptual model to predict Web users actions using a special ontology for the task. In is notable that their work addressed anonymous but yet profiled Web users. With the growing importance of CPSS, need prediction and personalization of provided services also gains its increasing share for such systems. Zheng et al. (2018) explored the predictability of passenger traffic flows for better group elevator dispatching using machine learning to analyze historical data. To accommodate the passenger arrival as a stochastic process, dynamic time warping with $K$-means clustering is used to measure similarity of traffic data. The data is then used to find an optimal elevator group dispatching scheme minimizing wait and travel time for passengers. Asad et al. (2020) proposed a machine-learning driven mobility prediction classification of travellers in the context of predicting safe travel times and routers for vulnerable age-groups. Machine-learning algorithms such as logistic regression, perceptron, support vector machine (SVM), $k$-nearest neighbour, and decision tree where tested, out of 
which SVM worked the best (classification accuracy over 80\%) for daily train travellers of London transport.

Compared to this earlier work, our work focuses on delivering a profiled passenger to a floor that suites her travelling needs the most, without any effort required from the passenger, i.e., no need to use the switch panel in the elevator car to dial the destination floor. To the best of our knowledge, personalization has not yet been applied in the context of smart elevator travels in such a way.

\section{Smart elevator system}

\subsection{Elevator system context}

The elevator system we use as a basis for the smart elevator is a conventional simple elevator system with a single car running in its allocated elevator shaft in a typical 8floor office building. The elevator is operated by push-button controls inside the car, and called to a floor by push-buttons (Up/Down travel, except Floors 0 and 7, where only one travel direction can be selected) located outside the elevator car on each floor. Figure 1 sets the system and building context. The building resides at university campus and hosts offices for university staff, labs, computer classes, and offices for private and start-up companies. The entrance floor of the building is Floor 1, whereas Floor 0 allows access to a secured parking area (only a number of car-owners with a special permit have access to the parking area, yet everyone can take an elevator to Floor 0 as well), and maintenance rooms (cleaning services storage). Floors 1-7 are used for offices, Floors 1, 3, 4, 5, and 6 have also labs and computer classes, and Floors 2 and 7 are occupied by companies. Thereby, besides employees who have offices in the building, the regular visitors of this building are students. The travel needs of university academic staff and students are affected by the schedule and location of classes taking place, whereas for company employees such dependency is not expected to occur.

We now proceed to define the context of our elevator system. First, we define the context of a trip. An elevator call (a trip) is a ride between two floors, where at least one passenger is present in the elevator car. The elevator call is defined by its departure and destination floor, and the number of passengers on the call. During its moving the elevator car can serve multiple calls but only calls that are on its movement direction (e.g., if the elevator is moving downwards, it will make a stop on every floor where the call button for going down has been pressed). Herein, for the smart elevator, we do not consider empty calls - ferry trips without passengers - of interest. An elevator event is defined as any action a passenger takes with an elevator (e.g., voice command or a button press to travel to a desired floor), and events are logged in the smart elevator system. In the context of the smart elevator, floor predictions made by the system are also captured as additional events.

For the smart elevator model, we define the building to consist of $F$ floors such that $F=\left\{f_{0}, f_{1}, \ldots, f_{7}\right\}$, with a single elevator car $E$ running $U p$ or Down in its own shaft. Floor calls $C$ are initiated by passengers $P$ by pressing either the $U p$ or Down button indicating their desired travel direction. The elevator car makes a stop on the floor for which a call on its travel direction exists, or a travel destination has been selected by a 


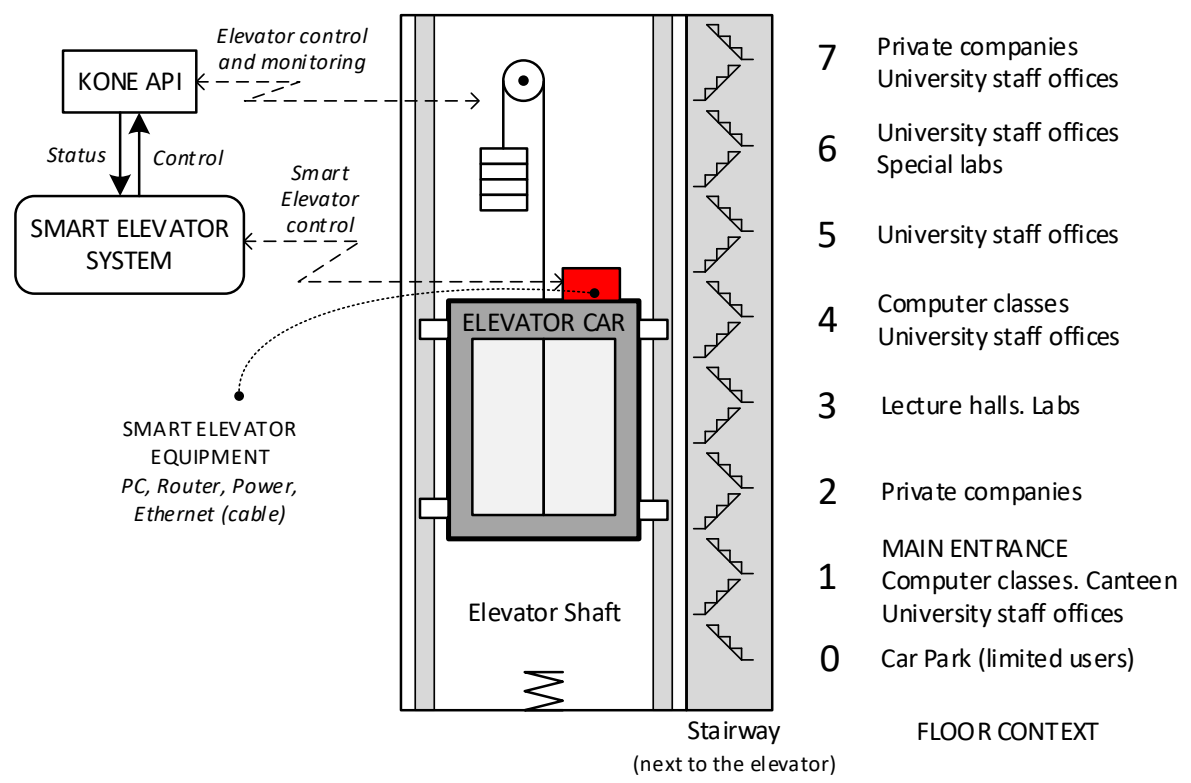

Fig. 1. Smart elevator system setup, and the building context.

passenger from the switch panel inside the car or declared via VUI. If the destination floor chosen by passenger is not on the car's travel direction, the elevator continues to serve calls on its travel direction before serving calls on the opposite direction.

\subsection{Smart elevator system architecture}

The smart elevator system is based on the existing conventional elevator built by KONE Corporation - a global leader in the elevator and escalator industry. Our building has two independent KONE elevators, each running in its own shaft. For the smart elevator, we use only one of these elevators - the elevator running in the North-shaft of the building. In addition to the existing elevator system, the following devices were installed in the elevator car to add the main features of the smart elevator (Figure 2):

- One Basler acA2040-25gc ${ }^{1}$ RGB camera with Ethernet communication, 4MP resolution, and global shutter. The camera is installed in the corner of the elevator facing the doors, and is able to capture video stream up to $25 \mathrm{fps}$ covering the area of elevator entrance and most of the cabin. The images captured (resolution $2048 \mathrm{x}$ 2048 px) by the camera are used as an input for face detection algorithm to detect human faces. The latter is based on HOG feature extractor (King, 2009) with linear

\footnotetext{
${ }^{1}$ https://www.baslerweb.com/en/products/cameras/area-scan-cameras/ace/aca2040-25gc/
} 


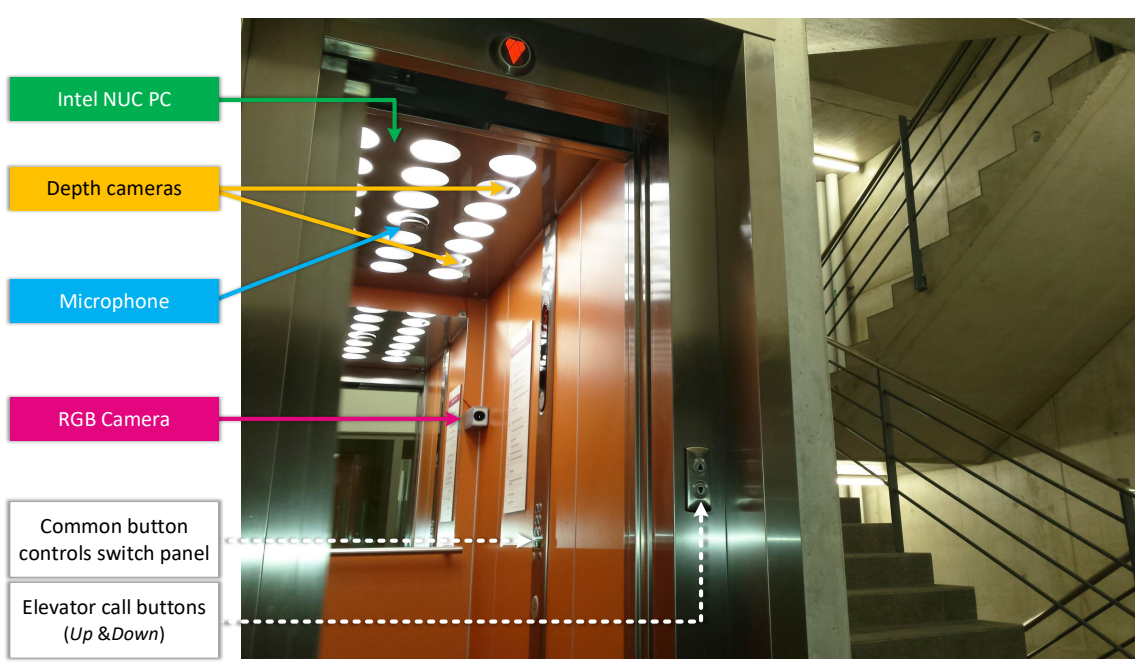

Fig. 2. Smart elevator system setup - a view into the elevator cabin.

classifier. Extracted face descriptions are then compared to existing face profiles using Radius Neighbour Classifier (Pedregosa et al., 2011) to detect and distinguish different passengers.

- Four Intel Real Sense D435² depth cameras with dual infrared light sensors located over the elevator ceiling cover. These are used to detect passenger movement and locations within the elevator cabin throughout the travel from the moment of entry until exiting the elevator car.

- One portable speakerphone Sennheiser SP20 $0^{3}$, which allows the passengers to control the elevator through voice commands using either Estonian or English as the command language. Currently, the passengers can request the elevator to take them to a specific floor (e.g., by giving the command Go to the first floor!), to a floor of an employee by stating employee name, and in addition ask about weather forecast and current date and time.

- An Intel NUC Mini PC NUC5i7RYB ${ }^{4}$ with 16 GB DDR3L RAM and 512GB NVMe SSD for processing elevator sensor data. This PC was placed on top of the elevator cabin ceiling.

The orchestration of different smart elevator components and subsystems is the responsibility of the smart elevator system Core (Figure 3), written in Python. In particular, the Core takes care of interacting with the KONE API (the elevator API) with the aim of making elevator calls and polling its status. The Core is also responsible for logging elevator event data, associating passenger identificators received through facial

\footnotetext{
${ }^{2}$ https://www.intelrealsense.com/depth-camera-d435/

${ }^{3}$ https://www.eposaudio.com/en/ee/enterprise/products/sp-20-ml-speakerphone-1000226

${ }^{4}$ https://www.intel.com/content/www/us/en/products/boards-kits/nuc.html?wapkw=nuc5i7ryb
} 
recognition, tracking passengers' entry and exit floors, predicting destination floors for passengers for automatic floor pre-selection and call, and lastly moving the elevator near floors with high volume of entry traffic while its idle. All interactions and events with the smart elevator are logged with timestamps added by the database management system (DBMS). We have used the PostgreSQL DBMS ver.11.4 for data storing. The operational database of the smart elevator contains 14 tables, which amongst operational details allow to store the following data:

- passenger profiles;

- passenger facial fingerprints;

- audio queries performed as voice commands to the elevator;

- passenger travel history together with departure and destination floor;

- elevator action log containing all smart elevator travels.

Regardless of the installed smart elevator features, the passengers of this smart elevator can still continue to manually operate it using the buttons on the switch panel in the elevator car, and select their desired destination floor. As our aim in this paper is the automatic passenger destination floor selection, we will limit our discussion on the system here.

Finally, in order to comply with privacy and security requirements, passengers of the smart elevator are informed about the existence of these devices in accordance with the law using notice boards near the elevator entrances. To preserve passengers privacy the captured biometric data is only stored as encoded feature vectors (not directly attributable to any particular person), and used on an anonymous basis by a machine learning algorithm. As only the North-elevator of the building is equipped with devices for smart elevator, the passengers are free to choose the second elevator - the Southelevator - in case of privacy concerns.

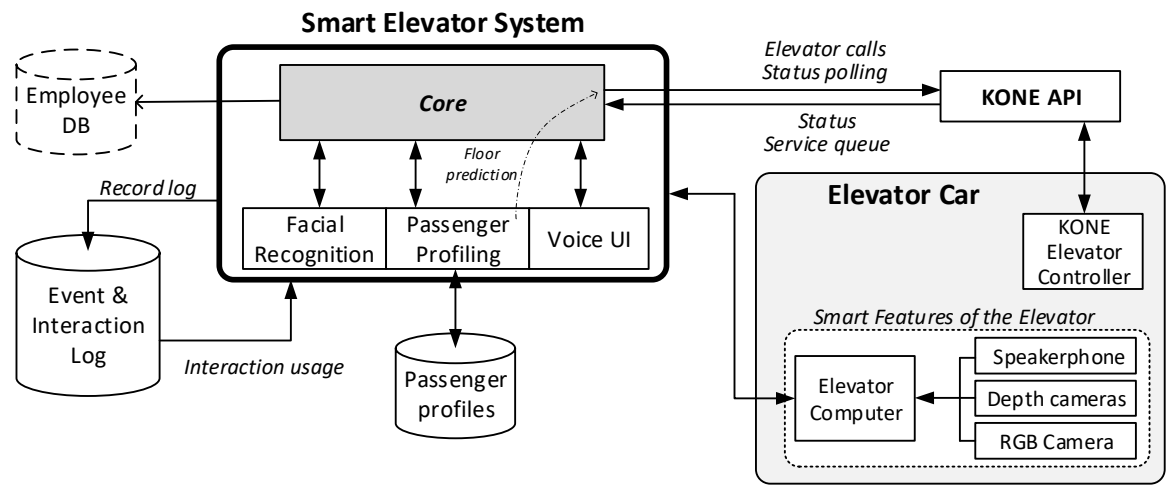

Fig. 3. Smart elevator system architecture. 


\subsection{Passenger profiles}

The identification of passengers is purely based on facial recognition using the facial fingerprints stored as 128-member vectors in the elevator system database. Each time a passenger enters the smart elevator, the system captures a video stream with the incar RGB camera, and facial recognition is carried out to detect whether the passenger already has a profile stored in the system or not. In case no match is found, a new passenger profile is created. The passenger profile consist of profile identificator, which distinguishes the passenger from others, and facial fingerprints, used to identify a passenger whenever she uses the elevator. These profiles are generated automatically by the system. No passenger profile is linked to an actual person with name and other data making her identifiable. With this approach, the smart elevator system operates around passengers, who's identity remains anonymous. The database of passenger profiles is created from scratch, and records of new profiles added upon arrival of new passengers (no match within existing profiles found). No prior-existing pre-configured database of passenger profiles is used.

Further, passenger profiles are connected to passengers' interactions history log, where an entry is made every time a passenger is successfully identified with facial recognition. This log links together profiled passengers with elevator interactions, and forms the basis for providing personalization and smart features for profiled passengers. We use the travel history to forecast passenger travel floor and make a personalized floor pre-selection for a profiled passenger. Presently, personalized floor pre-selection on elevator entry is provided for known (profiled) passengers, based on the most frequently travelled floor from a departure floor. Yet, this approach is not sufficient, and has provoked us to find ways to improve it through user profiling, as discussed in Section 4. During the test period of three months from November 2019 to February 2020, around 450 profiles were established.

\section{Towards personalized elevator travel}

In this section we address the personalized travel experience for profiled passengers. For passengers that remain unidentified by the system, the travel with the smart elevator remains conventional - they call the elevator, enter the elevator car, and select the desired destination floor from the button switch panel within the car. In addition, all passengers can use voice commands to set the destination floor over the VUI.

\subsection{Personalized travel}

One of the concepts of the smart elevator system is personalized approach towards passengers. Destination floor prediction is one of possible personalization services for elevators as CPSS. For passengers with existing profile of a particular building, commuting between two floors should be possible without a need to press any buttons inside the car - the elevator CPSS takes over this role. Once a profiled passenger enters the elevator and is identified, the system is able to predict the floor the passenger wants to reach, pre-selects the floor for the passenger and initiates the travel - all this without any need from a passenger to press buttons or interact in any other way with the 
elevator within the car, except to re-route the elevator in case of prediction mismatch. Consider the following possible scenario: a passenger enters the elevator car carrying some boxes and her hands are already occupied, the elevator knows where the passenger usually travels, and is able to help the passenger by automatically initiating a trip. Such floor prediction service could also be valuable for elderly (e.g., visually impaired) or disabled persons.

Presently, the smart elevator system predicts for a known traveller the most visited destination floor for a departure floor. For this, the smart elevator system pulls the passenger's travel history from the repository. The floor to which the passenger has travelled from the departure floor the most, is deemed to be the most probable destination floor. The precondition for this is that the floor has to have been visited by a passenger from the departure floor as a destination at least 10 times, in all other cases the departure-destination floor pair is not considered for the prediction. Thus, the smart elevator floor prediction system looks at floor pairs $F P$ such that $F P=f_{n} \times D$, where $f_{n} \in F$ and $D \subset F$ such that $f_{n} \notin D$, and $f_{n}$ is the departure floor and $D$ is a set of destination floors.

The feedback from the initial smart elevator test period indicated that the initial approach taken for floor predictions is not perceived useful, and in some cases even considered annoying. Thereby, we are now looking for ways to improve the system, including passenger context, travel behaviour patterns, also by time, and any other additional information to be included in anonymous passenger profiles to deliver better destination floor predictions, and quality of personalized elevator service.

Herein, we focus on anonymous passenger profiles for which we construct daily travel patterns. We then investigate, whether including these daily travel needs improves the present simple approach of destination floor prediction, and to what extent. To better understand passenger behaviour and travel needs, we first answer $\boldsymbol{R Q 1}$ to investigate the travel distances and likelihood of making short one-floor transits in Section 4.2. We then proceed to set the baseline for $\boldsymbol{R Q} 2$ and look at the extent the most travelled floor describes passenger travel behaviour (Section 4.3). This is followed by a study where we include time patterns (RQ3) for prediction improvement in Section 4.4. Finally, in Section 4.5 we explore the applicability of existing machine learning algorithms for destination floor prediction $(\boldsymbol{R Q 4})$ in the context of a smart elevator system.

\subsection{RQ1: Likelihood of short-distance transit}

The first question to consider is the likelihood of elevator passengers transiting only one floor. In particular, how likely an elevator is used just to travel one floor up or down. The motivation is to identify whether this aspect of travel behaviour could be used as a factor for floor prediction. If the hypothesis that passengers tend to prefer stairs to elevator to transit one floor holds true, this can be used as a valuable input for the decision process and should also be included in passenger profiles as a characteristic. This would help, for example in the case an equal or almost equal choice between two floors exists, to decide whether the long or short distance transit should be preferred for the floor prediction.

To answer $R Q 1$, we take a look on all the trips made with our smart elevator. Presently, our database holds data about 36,526 such trips collected over a time pe- 
riod of three months. These trips are the transits in between two floors without any intermediate stops. We do not consider 'ferry' trips - trips without any passengers - of value here.

In parallel, we also look at transits of profiled passengers - these however may contain several trips. The typical scenario for the latter case is that there are more than one passenger in the elevator car with different destination floors, or the elevator makes an intermediate stop to serve additional calls on its way. We look at passenger transits as these describe the real needs of elevator passengers for travel within a particular building. The passenger transits are formed based on the identified elevator passengers according to their profiles.

Figure 4 describes the results of this study. In case of individual trips, which also include intermediate stops, we see that in $16 \%$ of cases the elevator car moves only one floor up or down, while the majority of trips are either of distance of 3 or 4 floors. However, when considering the actual passenger travel needs, we see that only a small amount of travels $(6 \%)$ are between two floors on top of each other, and the majority of travels are derived by the need to transit between several floors. Thereby, we conclude that travel distance information should be included in passenger profiles to personalize the floor prediction decision for a particular passenger.

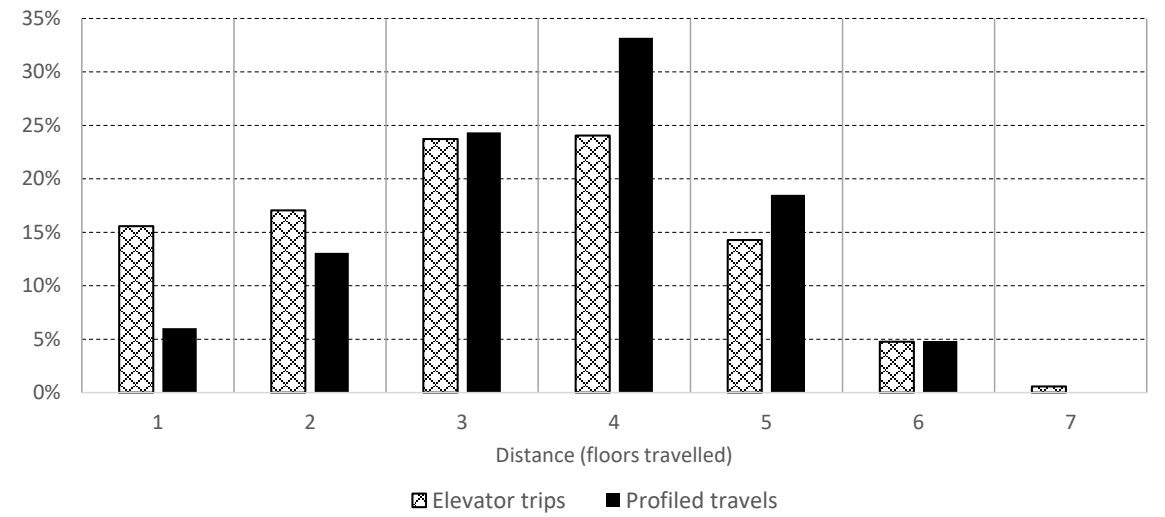

Fig. 4. Elevator car travel distances for trips, and passenger travel needs by distance.

The findings also confirm our hypothesis on passengers' travel behaviour. Evidently, taking stairs to transit one floor up or down suits for most of the visitors and inhabitants of the building. It is highly likely that this is due to the fact that taking one floor by stairs is generally faster than waiting for the elevator to take the same trip. It should be also noted that the stairs in our building are just next to the elevator (Figure 1) without any need to pass any additional doors or walk to the stairs. We believe this to be a contributing factor for elevator travellers' behaviour to prefer stairs over waiting - the closeness and easy access makes stairs attractive. In buildings, where stairs are 
further away, or behind fire doors, passengers behaviour might be different. The waiting time problem has been a source for many research works (Bharti et al, 2017; Chou et al. 2018; Fujimura et al., 2013), yet not addressing the attractiveness of stairs.

We acknowledge that these findings apply to the particular building type - the building where our smart elevator is located, and are also somewhat dependant on the floor context. Even though for another type of building these findings might be different, some of the conclusions can still be generalized. For our smart elevator system, the results provide a valuable input for floor prediction improvement, and confirm the necessity to include this knowledge into prediction algorithm and passengers' profiles for providing personalized elevator travel.

\subsection{RQ2: Frequent destination floor as prediction Basis}

The second question we consider is to what extent does the most frequently travelled floor describe passengers' behaviour and travel needs. In an 8-floor building, the probability to travel from one floor to any other floor is theoretically equal, and can be expressed as $p=1 /(8-1)=14.3 \%$. Obviously, this is purely theoretical and does not count the context of the floor, and the passenger's travel habits and needs.

We now proceed to investigate whether using the most travelled floor from the passenger's profile is more accurate than assuming that the passenger takes one of the floors randomly. For this we investigated the interaction logs for profiled passengers. After data cleaning we ended up with only 497 trips for which the prediction data was correctly available, due to the malfunction of the KONE API. Unfortunately, KONE API is beyond our authorized capabilities.

Table 1 describes the results of the study. Here the profiled trip is defined as a travel of a profiled passenger between two floors (even if the elevator car made intermediate stops) for which the smart elevator system proposed a predicted destination floor. As observed, there is a great variability of $30 \%$ in prediction accuracy over different profiles - apparently some profiled passengers have more fixed travel behaviour (need to transit in between floors) than others. As the results indicate, the approach of using the most frequently travelled floor for prediction is approximately four times more accurate than just randomly picking a floor to predict. Interestingly, the approach has better accuracy for downwards travel (to entrance floor) than for travels going upwards. We believe this to be caused by the typical travel pattern of departing from higher floors to entry/exit floor, and as the logs confirm, the entrance floor is the most travelled one in passenger profiles. It should also be noted that not all the travels of known profiles were covered by the predictions (only 91 out of 453), as the minimum travel number to a floor as destination was not yet met (Section 4.1). All in all, the most frequently travelled floor cannot singly meet the need to reliably predict destination floor for known passengers, and thus it should be complemented with additional characteristics captured in passenger profiles to improve floor prediction accuracy.

\subsection{RQ3: The value of daily travel needs for destination floor prediction}

Next, we investigate passengers' travel needs and how such needs depend on daily activities expressed by time of day in a typical office building. We aim to show that the 
Table 1. Using frequently travelled floor for floor prediction for known passengers.

\begin{tabular}{|l|r|}
\hline Characteristic & Value \\
\hline \# trips with predictions & 497 \\
\# profiles covered & 91 \\
\# profiles total & 453 \\
\hline Average prediction accuracy for passenger profiles & $64.5 \%$ \\
\hline Average prediction accuracy over profiled trips & $73.1 \%$ \\
Average prediction accuracy over profiled trips (downwards travel) & $59.6 \%$ \\
Average prediction accuracy over profiled trips (upwards travel) & $65.7 \pm 30.2 \%$ \\
\hline
\end{tabular}

inclusion of daily travel patterns, e.g, going for a lunch in the canteen on the first floor, or outside the building, will improve the accuracy of the present simple floor prediction approach. As a baseline we use already collected prediction data on real elevator travels for known passengers.

To answer the question whether the inclusion of travel needs set off by daily activities would improve floor prediction, we first run a simulation on collected travel data on known passengers, and study if including the time of travel with a precision of an hour improves floor prediction (Figure 5).

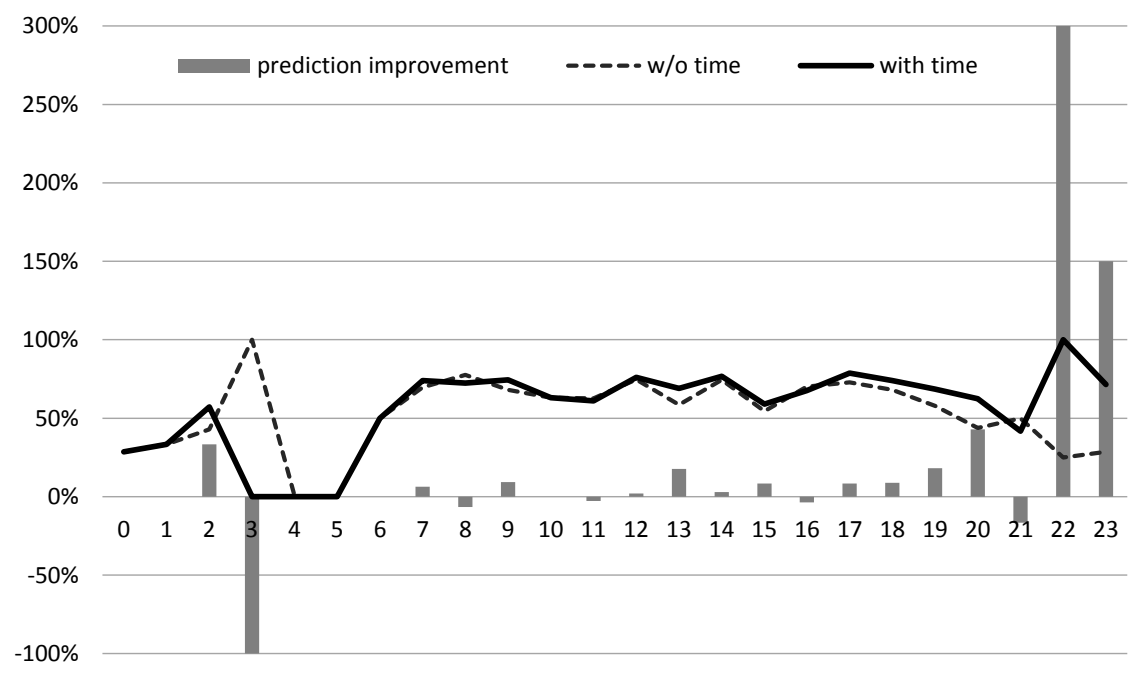

Fig. 5. Destination floor prediction accuracy for known travellers without considering travel time (present prediction implementation), considering time of travel, and prediction improvement gained on a 24-hour scale.

Although the simulation showed that including the time of travel slightly improves (+6.7\% improvement, where the average accuracy without considering time is at $53.1 \%$ 
and considering the time of travel at $56.6 \%$ ) destination floor prediction for known passengers, this is not always the case, and some results were conflicting. The deterioration of prediction accuracy for 3 a.m. (Figure 5) is based only on two travels, whereas the improvement for 10 p.m. and 11 p.m. are based respectively on the data of four and seven travels; for all other hours more than 20 travels were available. For the night hours 4 a.m. and 5 a.m. there was no travel data available. The collected data highlighted that on one hand, travels are not always happening with a precision of an hour, e.g., a person may come to work at 7:50 a.m. classified as hour 7, or ten minutes later at 8:00 a.m. classified as hour 8 - thus hourly granulation might be too specific to express passengers travel habits on a time scale. On the other hand, travel logs on hourly travels show particular peaks and slopes in travel needs with the elevator (Figure 6).

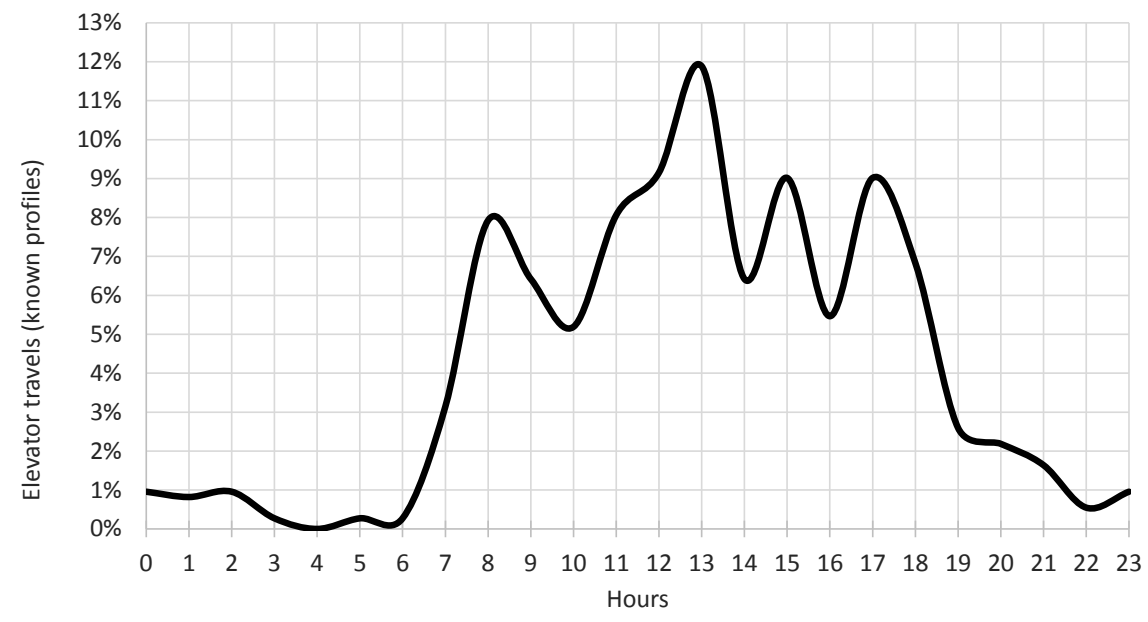

Fig. 6. Travel need distribution by hours.

Considering that hourly granulation of data is too specific, and there are clear peaks and slopes in travel needs, we proceed to define travel activity context of time through four separate time periods (slots) throughout the working day, starting of 6 a.m. (Table 2), with period start time inclusive and end time exclusive.

Re-running the simulation on the four defined time slots indicates that lessening time granularity of 24 hours to only four slots constructed based on travel needs (peaks and slopes on Figure 6) to outperform the previous travel time approach $(+7.9 \% \mathrm{im}-$ provement). Table 2 compares the results of prediction accuracy of the present simple approach of using the most travelled floor (col. 3) to floor prediction made based on the passengers' travel history for particular time context (col.4). As indicated, there is no particular improvement for predictions made for morning arrivals - passengers arrive and depart to upper floors, however a small improvement of ca $6 \%$ can be observed for predictions in the Midday and Leaving context. Thus, during morning arrival time 
Table 2. Using frequently travelled floor for floor prediction for known passengers.

\begin{tabular}{|l|l|r|r|r|}
\hline $\begin{array}{l}\text { Period of day } \\
(24 \mathrm{~h})\end{array}$ & Context & $\begin{array}{r}\text { Prediction } \\
\text { accuracy [\%] }\end{array}$ & $\begin{array}{r}\text { Periodic prediction } \\
\text { accuracy [\%] }\end{array}$ & $\begin{array}{r}\text { Improvement } \\
{[\%]}\end{array}$ \\
\hline $06-11$ & Arrival & 70.2 & 70.8 & 0.8 \\
$11-15$ & Midday/Lunch & 66.5 & 70.4 & 5.8 \\
$15-19$ & Leave & 65.8 & 69.8 & 6.2 \\
$19-06$ & 'Night-owls' & 43.9 & 54.9 & 25.0 \\
\hline \multicolumn{2}{|l}{} & 61.6 & 66.5 & 7.9 \\
\hline
\end{tabular}

slot passengers' behaviour is quite regular, and for the rest of the day dependent on different daily activities causing the need to travel with the elevator. Interestingly, an improvement of $25 \%$ is found for 'Night-owls', for reasons previously explained.

In summary, including the daily travel need patterns of known users by period of time into floor prediction has the potential to improve the prediction accuracy and provide a better personalization. Yet, the granularity of 24 hours is too specific to capture and describe these patterns, although providing prediction improvement. Therefore, it is rational to base the travel time patterns on rather volatile predefined time slots identifiable from passengers' travel history. We conclude that time of travel information must be included for floor prediction, and passenger profiles extended to indicate daily travel needs by time.

\subsection{RQ4: Applicability of existing algorithms for destination floor prediction}

Having explored passengers' travel behaviour and travel needs in the context of smart elevator, and having shown that travel behaviour provides additional characteristics to the existing simple prediction approach, we now turn to machine learning (ML) algorithms, to investigate their applicability for the task. In particular, we experiment with the perceptron algorithm (Minsky and Papert, 1972), $k$-nearest-neighbour (KNN) (Cover and Hart, 1967), logistic regression (LR) (Cramer, 2003), support vector machine (SVM) (Cortes and Vapnik, 1995; Ben-Hur et al. 2001), and decision tree (DT) (Quinlan, 1987) approaches, and use them to simulate floor prediction.

All the chosen algorithms aim to solve a classification problem. The classification model is the simplest type of predictive analytic models, where data is categorized based on historical data. Each of these algorithms has different behaviour. We experiment with the selected set of algorithms to compare the algorithms and find the best suitable one for our floor prediction problem. The same dataset as in $R Q 3$ is used to simulate floor predictions using the ML algorithms. We distribute the data into two subsets - the first $80 \%$ of data (as generated by passengers travels by time) we use to train the models, and the rest $20 \%$ we use to validate the result. The results of the ML algorithms are compared to the present simple floor prediction approach used as a baseline. Table 3 presents the results.

The perceptron algorithm is based on linear prediction function. The experiments (Table 3) show that this algorithm does not achieve good results, implying that the elevator floor prediction should be described as a non-linear function. The LR algorithm 
could be well regularized, and would provide better results with more training data being used. The KNN algorithm is one of the most fundamental non-parametric methods used for classification and regression, which works well with small dataset. The SVM algorithm - one of the most robust prediction methods - works well for data not linearly separable, and guarantees overfitting. The DT approach, which is non-parametric, easy handle feature interactions, provides the best precision among all other algorithms; however it is very sensitive to new data. Overall, all the algorithms in this experiment somewhat suffer from the small size of the dataset we have used, becoming especially critical when data of every profiled passenger is separated by a period of the day - in every category the amount of data is even smaller. Yet, the initial experiment with ML algorithms shows that KNN and DT have the potential to contribute for floor prediction.

Table 3. Comparison of ML algorithms accuracy [\%] for floor prediction.

\begin{tabular}{|l|r|r|r|r|r|r|}
\hline Type & Baseline & Perceptron & LR & KNN & SVC & DT \\
\hline Overall (w/o period grouping) & 64.6 & 41.5 & 51.7 & 61.2 & 51.7 & 66.0 \\
\hline Period of day (24h) & & & & & & \\
$06-11$ & 70.2 & 52.2 & 69.6 & 73.9 & 65.2 & 78.3 \\
$11-15$ & 66.5 & 37.5 & 40.6 & 28.1 & 40.6 & 40.6 \\
$15-19$ & 65.8 & 42.9 & 50.0 & 46.4 & 39.3 & 58.9 \\
$19-06$ & 43.9 & 27.8 & 58.3 & 66.7 & 50.0 & 58.3 \\
\hline
\end{tabular}

We now set forward a hypothesis - what if we could provide the ML algorithms the information about travel direction, and explore the effect including this information has on the floor prediction. The travel direction could be acquired through the elevator call from KONE API - remember the elevator had 2 call buttons ( $U p \&$ Down) on each floor, except Floors 0 and 7 . From the existing data we are able to derive the travel direction, and run another set of simulations with ML algorithms (Table 4). For the baseline we rely on existing predictions, which do not consider travel direction.

Table 4. Comparison of ML algorithms accuracy [\%] for floor prediction while considering travel direction as an additional input parameter.

\begin{tabular}{|l|r|r|r|r|r|r|}
\hline Type & Baseline & Perceptron & LR & KNN & SVC & DT \\
\hline Overall (w/o period grouping) & 64.6 & 38.1 & 53.7 & 64.6 & 53.7 & 70.1 \\
\hline Period of day (24h) & & & & & & \\
$06-11$ & 70.2 & 56.5 & 82.6 & 91.3 & 82.6 & 91.3 \\
$11-15$ & 66.5 & 31.3 & 40.6 & 40.6 & 40.6 & 46.9 \\
$15-19$ & 65.8 & 44.6 & 50.0 & 50.0 & 46.4 & 62.5 \\
$19-06$ & 43.9 & 55.6 & 55.6 & 66.7 & 52.8 & 61.1 \\
\hline
\end{tabular}

As shown (Table 4), including travel direction improves the prediction accuracy for ML algorithms, except for the perceptron. Introducing a new additional feature - travel direction - on one hand helps to reduce the amount of classification options, and on the 
other hand travel direction is not dependable from other features. Thus, it narrows down selection possibility and improves prediction. The trend however remains the same, the two best algorithms are KNN and DT.

All in all, the elevator floor prediction problem is far from linear, and based on the collected data we know that KNN and DT are the most potential approaches to be used for floor prediction either seldom or in combination with some other approach. The experiments also highlighted that the ML algorithms need a large set of data for prior training.

\section{Conclusions}

With the advancement of technology and increasing computerization, cyber-physical social systems (CPSS) are gaining their importance in our daily lives in the role of supporting and assisting our activities. In this paper, we have explored a possibility to provide personalized travel experience for passengers of a smart elevator system - a cyber-physical social system. Of particular interest was to investigate how to improve the existing destination floor prediction, currently based simply on the most frequently visited floor. For this we investigated passengers' travel needs and behaviour within a 8-story office building where the smart elevator system was set up on a conventional single-car single-shaft KONE elevator - a type of elevator that can be found in many buildings. The smart elevator approach described in this article could also be applicable for other types of buildings with regular visitors or permanent inhabitants, e.g., apartment buildings, hotels, and hospitals.

Our research on elevator passenger behaviour indicates that well-located stairs next to elevator are attractive and passengers are likely to take stairs instead of travelling a distance of one floor by an elevator, as this saves time on waiting for the elevator and transiting to adjacent floor. The finding can be used as a valuable factor in floor prediction algorithm. Next, we explored the extent the most frequently travelled floor describes passengers' travel needs. The study results indicate that even though the most frequently travelled floor gives suggestions for predictions, it is not merely enough, and additional information describing passenger behaviour and travel needs must be included in passenger profiles for better destination floor prediction accuracy. Such information could be the time context of daily activities. As the study showed, including daily travel needs and time context can provide lift for the floor prediction accuracy. Further, the experiments with five different machine learning algorithms showed that the elevator floor prediction problem is clearly non-linear, and if used the algorithms need a large quantity of training data for precision. Including additional features such as the indicative travel direction, can contribute to prediction accuracy. It is proposed that the $k$-nearest neighbour algorithm and decision trees are the most suitable machine learning algorithms for floor prediction. The study was limited to anonymous passenger profiles - passengers were profiled as anonymous units based on real-time facial recognition, whereas no personal data or prior knowledge was used.

For the future we continue collecting interaction data from the smart elevator system, and experiment with various approaches and settings for the smart elevator concept. One of the tracks we continue working on, is improving destination floor pre- 
diction. It would be interesting to simulate predictions with combinations of different prediction approaches with different weights. In further, we would like to have the elevator to confirm the predicted floor with a passenger over voice user interface, allowing the passenger to overwrite the predicted floor with another destination floor, as necessary. We see a potential in learning from this as well for improving the predictions. Also, of interest is to study how the schedule of different classes affects passengers' (typically students) travel needs in the building, and how this information could be used to minimize elevator waiting time. With the emergence of the need for contactless and safer elevator travel (due to the still ongoing COVID-19 pandemic), we are also exploring the options of achieving the latter through a special configuration of CPSS. And lastly, what of personal information would passengers be willing to provide to get personalized elevator service.

\section{References}

Adomavicius, G., Tuzhilin, A. (2005). Toward the next generation of recommender systems: a survey of the state-of-the-art and possible extensions. IEEE Transactions on Knowledge and Data Engineering. 17(6), 734-749.

Allen, J. (2003). Speech Recognition and Synthesis. Encyclopedia of Computer Science. John Wiley and Sons Ltd. GBR, 1664-1667.

Asad, S. M., Dashtipour, K., Hussain, S., Abbasi, Q. H., Imran, M. A. (2020). Travelers-Tracing and Mobility Profiling Using Machine Learning in Railway Systems. 2020 International Conference on UK-China Emerging Technologies (UCET), Glasgow, United Kingdom, 14.

Bamunuarachchi, D.T., Ranasinghe, D.N. (2015). Elevator group optimization in a smart building. 2015 IEEE 10th International Conference on Industrial and Information Systems (ICIIS), IEEE, 71-76.

Ben-Hur, A., Horn, D., Siegelmann, H., Vapnik, V.N. (2001). Support vector clustering. Journal of Machine Learning Research. 2, 125-137.

Bharti, H., Saxena, R.K., Sukhija, S., Yadav, V. (2017). Cognitive model for smarter dispatch system/elevator. 2017 IEEE International Conference on Cloud Computing in Emerging Markets (CCEM), IEEE, 21-28.

Brand, M., Nikovski, D. (2004). Optimal parking in group elevator control. IEEE International Conference on Robotics and Automation, 2004. Proceedings. ICRA '04. vol. 1, 1002-1008.

Brocken, E., Hartveld, A., de Koning, E., van Noort, T., Hogenboom, F., Frasincar, F., Robal, T. (2019). Bing-CF-IDF+: A semantics-driven news recommender system. In: Giorgini, P., Weber, B. (Ed.) Advanced Information Systems Engineering, Springer International Publishing, Cham, 32-47.

Cassandras, C.G. (2016). Smart cities as cyber-physical social systems. Engineering. 2(2), 156158.

Cernys, P., Kubilius, V., Macerauskas, V., Ratkevicius, K. (2003). Intelligent control of the lift model. Proc. Second IEEE International Workshop on Intelligent Data Acquisition and Advanced Computing Systems: Technology and Applications, IEEE, 428-431.

Chou, S., Budhi, D.A., Dewabharata, A., Zulvia, F.E. (2018). Improving elevator dynamic control policies based on energy and demand visibility. 2018 3rd International Conference on Intelligent Green Building and Smart Grid (IGBSG), IEEE, 1-4.

Cortes, C., Vapnik, V. (1995). Support-vector networks. Machine Learning. 20(3), 273-297.

Cover, T., Hart, P. (1967). Nearest neighbor pattern classification. IEEE Transactions on Information Theory. 13(1), 21-27. 
Cramer, J. S. (2003). The Origins of Logistic Regression. Tinbergen Institute Working Paper No. 2002-119/4. SSRN Electronic Journal.

Dewen, Z., Li, J., Yuwen, Z., Guanghui, S., Kai, H. (1997). Modern elevator group supervisory control systems and neural networks technique. 1997 IEEE Intl Conference on Intelligent Processing Systems, (Cat.No.97TH8335), vol. 1, IEEE, 528-532.

Ding, N., Chen, T., Luh, P.B., Zhang, H. (2014). Optimization of elevator evacuation considering potential over-crowding. Proceeding of the 11th World Congress on Intelligent Control and Automation, IEEE, 2664-2668.

Dressler, F. (2018). Cyber physical social systems: Towards deeply integrated hybridized systems. 2018 International Conference on Computing, Networking and Communications (ICNC), Maui, HI, IEEE, 420-424.

Eguchi, T., Hirasawa, K., Jinglu Hu, Markon, S. (2004). Elevator group supervisory control systems using genetic network programming. Proceedings of the 2004 Congress on Evolutionary Computation, (IEEE Cat.No.04TH8753), vol. 2, Portland, OR, IEEE, 1661-1667.

Eirinaki, M., Vazirgiannis, M. (2003). Web mining for web personalization. ACM Trans. Internet Technol. 3(1), 1-27.

Fernandez, J.R., Cortes, P. (2015). A survey of elevator group control systems for vertical transportation: A look at recent literature. IEEE Control Systems Magazine. 35(4), 38-55.

Fujimura, T., Ueno, S., Tsuji, H., Miwa, H. (2013) Control algorithm for multi-car elevators with high transportation flexibility. 2013 IEEE 2nd Global Conference on Consumer Electronics (GCCE), Tokyo, IEEE, 544-545.

Gath-Morad, M., Schaumann, D., Zinger, E., Plaut, P. O., Kalay, Y. E. (2017). How Smart is the Smart City? Assessing the Impact of ICT on Cities. In: M.-R. Namazi-Rad, L. Padgham, P. Perez, K. Nagel, A. Bazzan (Eds.), Agent Based Modelling of Urban Systems, Vol. 10051, Springer International Publishing, 189-207.

Gauch, S., Chaffee, J., Pretschner, A. (2003). Ontology-based personalized search and browsing. Web Intelligence and Agent Systems. 1(3-4), 219-234.

Gaudioso, E., Boticario, J.G. (2003). User modeling on adaptive web-based learning communities. In: Palade, V., Howlett, R.J., Jain, L. (Eds.) Knowledge-Based Intelligent Information and Engineering Systems, Springer, 260-266.

Ge, H., Hamada, T., Sumitomo, T., Koshizuka, N. (2018). Intellevator: A context-aware elevator system for assisting passengers. 2018 IEEE 16th International Conference on Embedded and Ubiquitous Computing (EUC), Bucharest, IEEE, 81-88.

Ge, H., Hamada, T., Sumitomo, T., Koshizuka, N. (2018). Precaelevator: Towards zero-waiting time on calling elevator by utilizing context aware platform in smart building. 2018 IEEE 7th Global Conference on Consumer Electronics (GCCE), Nara, 566-570.

Goetsu, S., Sakai, T. (2019). Voice input interface failures and frustration: Developer and user perspectives. The Adjunct Publication of the 32nd Annual ACM Symposium on User Interface Software and Technology (UIST '19), Association for Computing Machinery, New York, NY, USA, 24-26.

Hikita, S., Iwata, M., Abe, S. (2004). Elevator group control with destination call entry and adaptive control. IEEJ Transactions on Electronics, Information and Systems. 124(7), 1471-1477.

Hou, J., Qiu, R., Xue, J., Wang, C., Jiang, X.-Q. (2020). Failure Prediction of Elevator Running System Based on Knowledge Graph. Proceedings of the 3rd International Conference on Data Science and Information Technology, 53-58.

ISO. (2012). ISO 18738-1:2012 Measurement of ride quality — Part 1: Lifts (elevators). International Organization for Standardization (ISO).

ISO. (2020). ISO 8100-32:2020 Lifts for the transportation of persons and goods - Part 32: Planning and selection of passenger lifts to be installed in office, hotel and residential buildings. International Organization for Standardization (ISO). 
Ketkar, S.S., Mukherjee, M. (2011). Speech recognition system. Proceedings of the Intl Conference \& Workshop on Emerging Trends in Technology, ICWET'11, Association for Computing Machinery, New York, NY, USA, 1234-1237.

Kim, J.-H., Moon, B.-R. (2001). Adaptive elevator group control with cameras. IEEE Transactions on Industrial Electronics. 48(2), 377-382.

King, D.E. (2009) Dlib-ml: A machine learning toolkit. J. Mach. Learn. Res.. 10, 1755-1758.

Kwon, O., Lee, E., Bahn, H. (2014). Sensor-aware elevator scheduling for smart building environments. Building and Environment. 72, 332-342.

Lee, E.A., Seshia, S.A. (2016). Introduction to Embedded Systems: A Cyber-Physical Systems Approach. The MIT Press, 2nd edn.

Liang, C.J.M., Tang, J., Zhang, L., Zhao, F., Munir, S., Stankovic, J.A. (2013). On human behavioral patterns in elevator usages. Proceedings of the 5th ACM Workshop on Embedded Systems For Energy-Efficient Buildings, BuildSys'13, Association for Computing Machinery, New York, NY, USA, 1-2.

Luo, F., Xu, Y. -G., Cao, J. -Z. (2005) Elevator traffic flow prediction with least squares support vector machines. 2005 International Conference on Machine Learning and Cybernetics, vol. 7. Guangzhou, China, 4266-4270.

Marinov, M. B., Nikolov, D. N., Ganev, B. T., Djamiykov, T. S. (2020). Smart Multisensor Node for Remote Elevator Condition Monitoring. 21st International Symposium on Electrical Apparatus \& Technologies (SIELA), Bourgas, Bulgaria, 1-4.

Meenatchi, D., Aishwarya, R., Shahina, A. (2016). A Voice Recognizing Elevator System. In: L. P. Suresh, B. K. Panigrahi (Eds.), Proceedings of the International Conference on Soft Computing Systems, Vol. 397, Springer India, 179-187.

Minsky, M., Papert, S. A. (1972). Perceptrons: An introduction to computational geometry (2. print. with corr). The MIT Press.

Olalere, I. O., Dewa, M., Nleya, B. (2018). Remote Condition Monitoring of Elevator's Vibration and Acoustics Parameters for Optimised Maintenance Using IoT Technology. 2018 IEEE Canadian Conference on Electrical \& Computer Engineering (CCECE), Quebec City, QC, $1-4$.

Pearl, C. (2017). Designing voice user interfaces: Principles of conversational experiences. O'Reilly.

Pedregosa, F., Varoquaux, G., Gramfort, A., Michel, V., Thirion, B., Grisel, O., Blondel, M., Prettenhofer, P., Weiss, R., Dubourg, V., et al. (2011). Scikit-learn: Machine learning in Python. J. Mach. Learn. Res.. 12, 2825-2830.

Quinlan, J. R. (1987). Simplifying decision trees. International Journal of Man-Machine Studies. 27(3), 221-234.

Reinsalu, U., Robal, T., Leier, M. (2020). Floor Selection Proposal for Automated Travel with Smart Elevator. Databases and Information Systems, Communications in Computer and Information Science (CCIS), vol. 1243, Springer, Cham., 38-51.

Robal, T., Kalja, A. (2010). Conceptual web users actions prediction for ontology-based browsing recommendations. Information Systems Development: Towards a Service Provision Society, Springer US, Boston, MA, 121-129.

Robal, T., Zhao, Y., Lofi, C., Hauff, C. (2018). Webcam-based attention tracking in online learning: A feasibility study. 23rd International Conference on Intelligent User Interfaces (IUI '18), ACM, New York, NY, USA, 189-197.

Ross, S., Brownholtz, E., Armes, R. (2004). Voice user interface principles for a conversational agent. Proceedings of the 9th International Conference on Intelligent User Interfaces, IUI '04, Association for Computing Machinery, New York, NY, USA. 364-365.

Russell, S., Norvig, P. (2009). Artificial Intelligence: A Modern Approach. Prentice Hall Press, USA, 3rd edn. 
Sieg, A., Mobasher, B., Burke, R.D. (2007). Learning ontology-based user profiles: A semantic approach to personalized web search. IEEE Intelligent Informatics Bulletin. 8(1), 7-18.

Silva, E.M., Boaventura, M., Boaventura, I.A.G., Contreras, R.C. (2018). Face recognition using local mapped pattern and genetic algorithms. Proceedings of the International Conference on Pattern Recognition and Artificial Intelligence (PRAI 2018), ACM, New York, NY, USA, $11-17$.

Skog, I., Karagiannis, I., Bergsten, A. B., Harden, J., Gustafsson, L., Handel, P. (2017). A Smart Sensor Node for the Internet-of-Elevators-Non-Invasive Condition and Fault Monitoring. IEEE Sensors Journal. 17(16), 5198-5208.

Speretta, M., Gauch, S. (2005). Personalized search based on user search histories. 2005 IEEE/WIC/ACM International Conference on Web Intelligence (WI 2005), IEEE Computer Society, 622-628.

Stark, L. (2019). Facial recognition is the plutonium of AI. XRDS. 25(3), 50-55.

Strang, T., Bauer, C. (2007). Context-aware elevator scheduling. 21st International Conference on Advanced Information Networking and Applications Workshops (AINAW'07), vol. 2, Niagara Falls, Ont., 276-281.

Turunen, M., Kuoppala, H., Kangas, S., Hella, J., Miettinen, T., Heimonen, T., Keskinen, T., Hakulinen, J., Raisamo, R. (2013). Mobile interaction with elevators: Improving people flow in complex buildings. Proceedings of Intl Conference on Making Sense of Converging Media, AcademicMindTrek' 13, ACM, New York, NY, USA, 43-50.

van Huijsduijnen, L. H., Hoogmoed, T., Keulers, G., Langendoen, E., Langendoen, S., Vos, T., Hogenboom, F., Frasincar, F., Robal, T. (2020). Bing-CSF-IDF+: A Semantics-Driven Recommender System for News. In J. Darmont, B. Novikov, R. Wrembel (Eds.), New Trends in Databases and Information Systems, Vol. 1259, Springer International Publishing, 143-153.

Wang, F., Tang, J., Zong, Q.: Energy-consumption-related robust optimization scheduling strategy for elevator group control system. 2011 IEEE 5th Intl Conference on Cybernetics and Intelligent Systems (CIS), Qingdao, 30-35.

Yaman, O., Karakose, M. (2017). Auto correlation based elevator rope monitoring and fault detection approach with image processing. 2017 International Artificial Intelligence and Data Processing Symposium (IDAP), Malatya, 1-5.

Zhao, W., Chellappa, R., Phillips, P.J., Rosenfeld, A. (2003). Face recognition: A literature survey. ACM Comput. Surv. 35(4), 399-458.

Zhao, H.C., Liu, X.Y. (2013). An improved DNA computing method for elevator scheduling problem. In: Zu, Q., Hu, B., Elçi, A. (Eds.) Pervasive Computing and the Networked World, Springer Berlin Heidelberg, Berlin, Heidelberg, 869-875.

Zheng, J., Tat Thomas, H. C., HuaiBing, Y. (2018). Traffic Prediction for Efficient Elevator Dispatching. TENCON 2018 - 2018 IEEE Region 10 Conference, Jeju, Korea (South), 22322236.

Zhuge, H. (2014). Cyber-physical society-the science and engineering for future society. Future Generation Computer Systems. 32, 180-186.

Received November 27, 2020 , accepted November 28, 2020 